\title{
Short-form Sun-style t'ai chi as an exercise training modality in people with COPD
}

\author{
Regina Wai Man Leung*,\#, Zoe J. McKeough", Matthew J. Peters ${ }^{\Uparrow}$ and Jennifer \\ A. Alison ${ }^{\#}$
}

ABSTRACT: The aims of the study were to determine the effect of short-form Sun-style t'ai chi (SSTC) (part A) and investigate exercise intensity of SSTC (part B) in people with chronic obstructive pulmonary disease (COPD).

Part A: after confirmation of eligibility, participants were randomly allocated to either the t'ai chi group or control group (usual medical care). Participants in the t'ai chi group trained twice weekly for 12 weeks. Part B: participants who had completed training in the t'ai chi group performed a peak exercise test (incremental shuttle walk test) and SSTC while oxygen consumption $\left(\mathrm{VO}_{2}\right)$ was measured. Exercise intensity of SSTC was determined by the per cent of $\mathrm{VO}_{2}$ reserve.

Of 42 participants (mean $\pm S D$ forced expiratory volume in $1 \mathrm{~s} 59 \pm 16 \%$ predicted), 38 completed part A and 15 completed part B. Compared to control, SSTC significantly increased endurance shuttle walk time (mean difference $384 \mathrm{~s}, 95 \% \mathrm{Cl}$ 186-510); reduced medial-lateral body sway in semi-tandem stand (mean difference $-12.4 \mathrm{~mm}, 95 \% \mathrm{Cl}-21-\mathbf{- 3}$ ); and increased total score on the chronic respiratory disease questionnaire (mean difference 11 points, $95 \% \mathrm{Cl} 4-18$ ). The exercise intensity of SSTC was $53 \pm 18 \%$ of $\mathrm{VO}_{2}$ reserve.

SSTC was an effective training modality in people with COPD achieving a moderate exercise intensity which meets the training recommendations.

KEYWORDS: Anxiety and depression, chronic obstructive pulmonary disease, exercise capacity, exercise physiology, health-related quality of life, rehabilitation

$\mathbf{T}$ 'ai chi, an exercise involving both upper and lower extremities, originated from China. T'ai chi is characterised by posture alignment, weight shifting and circular movements that incorporate elements of muscle endurance and strengthening, balance, relaxation and breathing control. The movements of $t^{\prime}$ ai chi are unique and are often described as slow, smooth flowing and graceful.

$\mathrm{T}^{\prime}$ ai chi has become a popular training mode in western countries and requires no exercise equipment or specific training venue and thus is easily implemented in the community. Currently, a number of t'ai chi styles are utilised, including Yang, Chen and Sun styles. Each of these styles is characterised by emphasis on slightly different forms (movements). The benefits of t'ai chi on balance, exercise capacity and muscle strength have been demonstrated in the healthy population $[1,2]$ and people with chronic conditions, such as chronic heart disease [3] and osteoarthritis [4].
In comparison with other styles of $t^{\prime}$ ai chi, shortform Sun-style t'ai chi (SSTC), also known as t'ai chi for arthritis, is a modified t'ai chi programme with 21 forms. This is one of the most common styles of t'ai chi and training courses for instructors are available worldwide. Each form can be broken down into several movements which are easy to teach and learn. Compared to some other styles of $\mathrm{t}^{\prime}$ ai chi, SSTC involves less difficult movements, such as less deep-knee bending and single leg standing, which may make it more suitable for older people.

Recent studies examining t'ai chi in people with chronic obstructive pulmonary disease (COPD) have shown conflicting findings on the effect of t'ai chi on exercise capacity and health-related quality of life (HRQoL) [5-7]. None of these studies examined the effect of SSTC in people with COPD. In addition, the exercise intensity of any style of $\mathrm{t}^{\prime}$ ai chi, including SSTC, in the COPD population is unknown. The current study had

\section{AFFILIATIONS}

*Dept of Physiotherapy, Concord Repatriation General Hospital Sydney,

${ }^{\#}$ Discipline of Physiotherapy, Faculty of Health Sciences, University of Sydney, Sydney, and

"Dept of Thoracic Medicine, Concord Repatriation General Hospital, Sydney, Australia.

\section{CORRESPONDENCE}

R.W.M. Leung

Dept of Physiotherapy, Concord Repatriation General Hospital

Hospital Road

Concord NSW 2139

Australia

E-mail: regina.leung@

sswahs.nsw.gov.au

Received:

March 012012

Accepted after revision: July 052012

First published online:

Aug 092012 
two parts. The aim of part A was to evaluate the effect of 12 weeks of SSTC on exercise capacity, balance, physical performance, quadriceps strength, HRQoL, anxiety, depression and self-reported difficulty in functional performance in people with COPD compared to no exercise training. The aim of part B was to investigate the exercise intensity of SSTC performance in people with COPD.

\section{METHODS \\ Part A}

Study design

A randomised trial was conducted with concealed allocation and blinded outcome assessment. The study took place at Concord Repatriation General Hospital, Sydney, Australia from June 2007 to October 2010. After confirmation of eligibility and collection of baseline measures, participants were randomly allocated to a t'ai chi group (TCG) or a control group (CG) via a computerised phone dial-up system. Random allocation with minimisation for lung function (forced expiratory volume in $1 \mathrm{~s}$ $>40 \%$ or $\leqslant 40 \%$ predicted), sex, and the main limiting symptom in the endurance shuttle walk test (ESWT) at baseline (i.e. dyspnoea, leg fatigue, or a combination of both symptoms) was used. Each participant maintained his/her medication regimen during the intervention period. The methodology of part A has been published previously [8]. An assessor, blinded to group allocation, performed the outcome measures at the beginning and end of the study. The study was conducted with the approval of the Sydney South West (Eastern Zone) ethics committee and the University of Sydney ethics committee. The trial was registered in Australian New Zealand Clinical Trials Registry (ACTRN12608000383369).

\section{Participants}

Participants were included if they had a diagnosis of COPD [9]. People were excluded if they had an acute exacerbation of COPD within the previous 4 weeks; had participated in formal exercise training in the previous 12 months; or had significant comorbidities including malignancy, symptomatic cardiovascular disease, other systemic or musculoskeletal disease that could hinder t'ai chi training; or required supplemental oxygen during training.

\section{Intervention}

Participants in the TCG attended two supervised, 1-h sessions, weekly for 12 weeks of SSTC training. Participants commenced training by learning two to three forms per week with completion of learning all 21 forms by week eight. In each training session, participants were trained with the SSTC forms that they had learnt up to and including that session. Each participant aimed to train at a moderate level of breathlessness or exertion, equating to a score of three on the modified Borg (0-10) scale [10]. To increase the training demand of SSTC, participants were asked to imagine pushing against resistance during all movements or to squat lower in certain movements. For participants whose dyspnoea or exertion did not reach the moderate level during training, wrist weights of between 0.5 and $1.5 \mathrm{~kg}$ were worn, with the weight determined by the individual's symptoms, although this is not common practice in t'ai chi training. Participants were also encouraged to focus on breathing rhythm, for example inhaling when the movements involved lifting, stepping forward or opening postures, and exhaling when movements involved lowering or closing postures of each t'ai chi movement. However, if the participant could not follow the breathing rhythm due to shortness of breath, a breathing rhythm that was comfortable for the participant was allowed. In addition to the two supervised training sessions each week, all participants in the TCG were asked to practice at home for $30 \mathrm{~min}$, on the other 5 days of the week. A t'ai chi training booklet and DVD (developed by the trainer) were provided to facilitate home training. Training duration was recorded by participants in the diary provided. Participants who wore wrist weights during the supervised training also wore the same weights for home training. All participants were trained by a physiotherapist with extensive experience in chronic lung disease management and who was an accredited SSTC trainer.

Participants in the CG continued with their usual medical care without any exercise training for 12 weeks after the baseline measurements.

Spirometry, lung volumes and diffusion capacity were measured at baseline according to standard protocols [11].

\section{Outcome measures}

All outcomes were measured at baseline (week 0) and study completion (week 12). The primary outcome measure was the endurance shuttle walk time. More detailed description of all outcome measures have been published previously [8].

\section{Exercise capacity}

Peak and endurance exercise capacity were measured by the incremental shuttle walk test (ISWT) [12] and ESWT [13], respectively. The scores of perceived exertion and dyspnoea were recorded before, each minute during and at the end of the tests using the modified Borg (0-10) scale [10]. The ISWT and the ESWT were performed twice at each testing occasion with at least $30 \mathrm{~min}$ rest between tests, with the better result used for analysis.

Physical performance, balance and quadriceps isokinetic strength Physical performance was measured by the modified physical performance battery test (MPPB) [14]. A score between 0-4 was generated with a higher score indicating better performance. Balance ability of participants was measured by the body sway tests [15] and functional reach test [16]. The quadriceps isokinetic strength was measured by the Kin Com (Isokinetic International, Chattanooga, TN, USA) with the speed set at 90 degrees per second.

\section{HRQoL, anxiety and depression and self-reported difficulty in functional performance}

HRQoL and anxiety and depression were measured by the Chronic Respiratory Disease questionnaire (CRQ) [17] and the Hospital Anxiety and Depression Scale (HADS) [18], respectively. Self-reported difficulty in functional performance was measured by the Functional Performance Inventory (FPI) [19].

\section{Compliance with SSTC training}

The compliance with the SSTC training was assessed by the total number of supervised sessions attended, the unsupervised home practice time per day and the number of days practised out of the five home training days per week. 


\section{Sample size}

Sample size calculation was based on detecting a minimum difference of $2 \mathrm{~min}$ [20] of endurance shuttle walk time between the TCG and the CG and used a baseline standard deviation of $1.8 \mathrm{~min}$ [21]. 42 participants (21 per group) were required in order to provide $90 \%$ power at a $5 \%$ significance level (two-sided) and allowing for a 15\% loss to follow-up.

\section{Data analysis}

Data analyses were conducted using SPSS version 17.0 (SPSS Inc., Chicago, IL, USA). Data are presented as mean \pm SD unless stated otherwise. All outcome measures were analysed using paired t-tests and repeated-measures analysis of variance with intention-to-treat analysis. A p-value of 0.05 (two-sided) was taken as the level of significance. The $95 \%$ confidence intervals around the mean differences were calculated. The scores of perceived exertion and dyspnoea in the ISWT and ESWT before and after the intervention were compared at isotime and at the end of exercise. Isotime was defined as the end time of the shorter pre- or post-intervention.

\section{Part B}

Study design

A repeated measures study design was used. Participants were those who had completed the 12-week SSTC training and who agreed to participate in this part of the study. Each participant completed an ISWT, rested for at least $30 \mathrm{~min}$ and then performed $15 \mathrm{~min}$ of SSTC. During the performance of SSTC, participants performed the 21 forms repeatedly for $15 \mathrm{~min}$. The assessment occurred within 2 weeks of completion of SSTC training. The measurements were only performed at the end of the training period when participants were fully competent in the performance of SSTC.

\section{Outcome measures}

Oxygen consumption $\left(\mathrm{VO}_{2}\right)$, carbon dioxide production $\left(\mathrm{VCO}_{2}\right)$, tidal volume, breathing frequency, minute ventilation were measured at rest, and during the ISWT and SSTC performance using a portable metabolic measurement system (Cosmed K4 $\mathrm{b}^{2}$; Cosmed, Rome, Italy). Oxygen saturation (Nonin 800R; Nonin Medical Inc., North Plymouth, MN, USA) and heart rate (Polar, Kempele, Finland) were also measured.

\section{Data analysis}

Physiological variables measured in the ISWT and the $15 \mathrm{~min}$ of SSTC performance were analysed, by averaging data from the last $25 \mathrm{~s}$ of each minute. The mean $V_{2}$ of SSTC performance $\left(\mathrm{VO}_{2}\right.$ (SSTC)) was determined by averaging the sum of $\mathrm{VO}_{2}$ each minute (from minute 2-15) during the SSTC performance. The intensity of SSTC was determined as a percentage of $V \mathrm{O}_{2}$ reserve. The $V_{\mathrm{O}}$ reserve was calculated as the difference between peak and resting $V_{\mathrm{O}_{2}}\left(V_{\mathrm{O}_{2}}\right.$ (ISWTpeak) $V \mathrm{O}_{2}$ (ISWTrest)) of the ISWT and the per cent $V \mathrm{O}_{2}$ reserve was calculated as: $\left(\left(V_{\mathrm{O}_{2}}(\mathrm{SSTC})-V \mathrm{O}_{2}\right.\right.$ (ISWTrest $\left.)\right) / V_{\mathrm{O}_{2}}$ reserve $) \times 100$. The minimal effective training intensity to improve cardiovascular fitness was considered as $30 \% \mathrm{VO}_{2}$ reserve [22]. The ISWT was chosen as the maximum exercise test in this study because evidence has shown that the ISWT elicits peak physiological responses in people with COPD [23]. The ESWT time of the participants in part B was compared to the CG using repeatedmeasures analysis of variance.

\section{RESULTS}

\section{Part A}

\section{Participant characteristics}

The flow of participants is presented in figure 1. 42 participants were recruited and 38 participants (90.5\%) completed the study with 19 in the TCG and 19 in the CG. All participants were Caucasian with no previous t'ai chi experience. Baseline characteristics of participants are presented in table 1 .

\section{Effect of intervention}

Results of exercise capacity, balance, physical performance, quadriceps strength, HRQoL, anxiety and depression and selfefficacy for the TCG and CG are presented in tables 2 and 3. The dyspnoea and rate of perceived exertion (RPE) scores in the ESWT at isotime are presented in table 2. Six participants in the TCG wore wrist weights ranging from 0.5 to $1.5 \mathrm{~kg}$ during training. At study completion, the ESWT time was significantly longer in the TCG than the CG. Compared to the CG, the TCG had a significant improvement in: incremental shuttle walk distance; body sway in anterior-posterior and medial-lateral direction in side by side stand and mediallateral sway in semi-tandem stand; functional reach distance; MPPB scores; quadriceps strength in both legs; CRQ scores in all domains; anxiety domain of the HADS and FPI score. At isotime, the RPE score during the ESWT was significantly less in the TCG compared to the CG.

Participants in the TCG attended mean \pm SD $21 \pm 2$ sessions out of a possible 24 sessions (91\%) and no adverse events were reported. Of the potential 5 days of unsupervised home

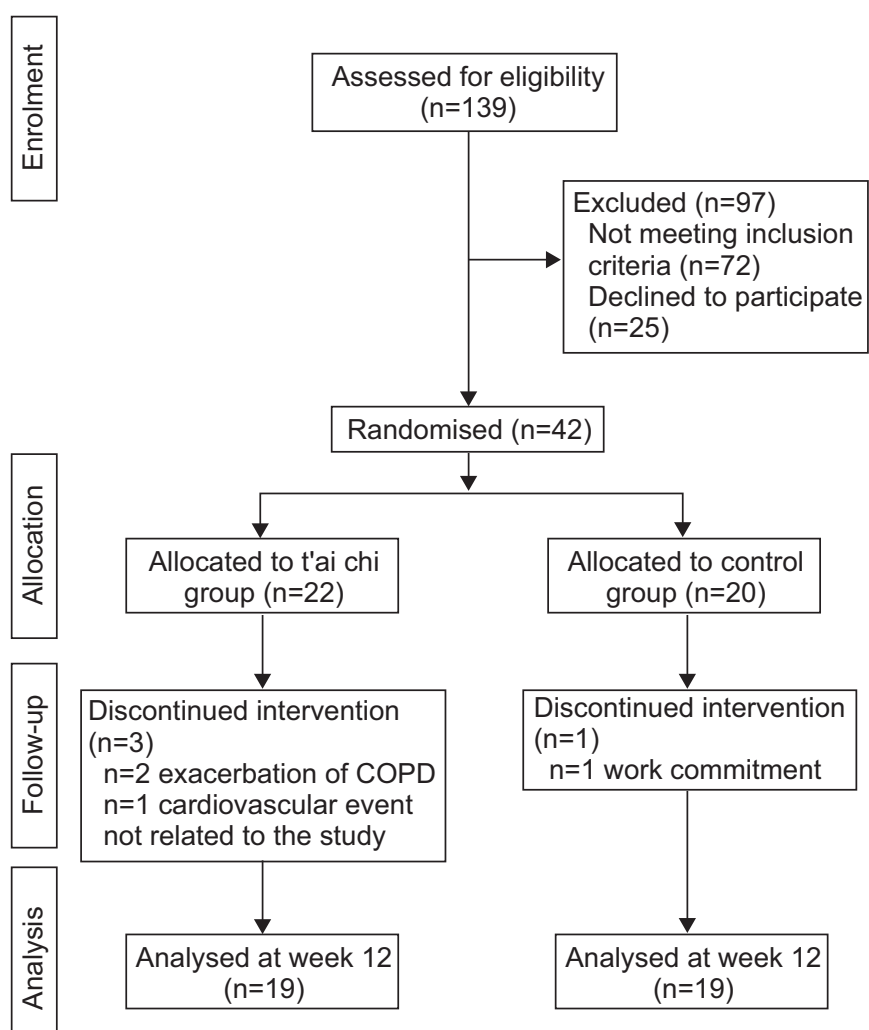

FIGURE 1. Flow diagram for part $A$ of the study. 


\begin{tabular}{|c|c|c|}
\hline Characteristics & Part A & Part B \\
\hline Participants n & 42 & 15 \\
\hline Age years & $73 \pm 8$ & $75 \pm 8$ \\
\hline Females & $15(36)$ & $8(53)$ \\
\hline Body mass index $\mathrm{kg} \cdot \mathrm{m}^{-2}$ & $27.4 \pm 5$ & $26.7 \pm 4$ \\
\hline FEV 1 L & $1.4 \pm 0.5$ & $1.4 \pm 0.4$ \\
\hline FEV $1 \%$ pred & $59 \pm 16$ & $63 \pm 14$ \\
\hline FVC L & $3.0 \pm 1$ & $3.0 \pm 1$ \\
\hline FVC \% pred & $102 \pm 23$ & $102 \pm 22$ \\
\hline 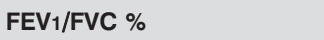 & $47 \pm 13$ & $50 \pm 15$ \\
\hline TLC \% pred & $103 \pm 16$ & $101 \pm 14$ \\
\hline FRC \% pred & $123 \pm 30$ & $113 \pm 33$ \\
\hline RV \% pred & $124 \pm 36$ & $118 \pm 34$ \\
\hline RV/TLC & $0.5 \pm 0.1$ & $0.5 \pm 0.1$ \\
\hline DLco \% pred & $55 \pm 16$ & $58 \pm 9$ \\
\hline \multicolumn{3}{|l|}{ Comorbidities } \\
\hline \multicolumn{3}{|l|}{$\%$ of total participants } \\
\hline Coronary heart disease & 33 & 13 \\
\hline Systemic hypertension & 55 & 27 \\
\hline Diabetes & 21 & 13 \\
\hline Dyslipidaemia & 38 & 13 \\
\hline Osteoarthritis & 60 & 73 \\
\hline Osteoporosis & 10 & 7 \\
\hline Absence of co-morbidities & 10 & 20 \\
\hline With 1 comorbidities & 14 & 27 \\
\hline With $\geqslant 2$ comorbidities & 76 & 53 \\
\hline
\end{tabular}

Data are presented as mean \pm SD or $n(\%)$, unless otherwise stated. FEV 1 : forced expiratory volume in $1 \mathrm{~s}$; FVC: forced vital capacity; TLC: total lung capacity; FRC: functional residual volume; RV: residual volume; $D$ LCO: diffusion capacity of the lung for carbon monoxide.

practice, participants practised for $4 \pm 1$ days per week for an average time of $27 \pm 10 \mathrm{~min}$ per day.

\section{Part B}

15 participants agreed to participate in part B of the study. All participants completed the assessment and their baseline characteristics are presented in table 1 . Six of these participants had worn wrist weights during SSTC training and also wore wrist weights during SSTC performance in this study. The physiological responses during the ISWT and SSTC performance are presented in table 4 . The per cent $V_{2}$ reserve of SSTC was $53 \pm 18 \%$. The ESWT time of the 15 participants was significantly greater than the CG from part A of the study (441 s, 95\% CI 255-613).

\section{DISCUSSION}

The novel findings of this study were that SSTC was more effective than usual medical care in improving exercise capacity, balance, physical performance, quadriceps strength, HRQoL, anxiety and self-efficacy compared to no exercise training in people with COPD. In addition, our study found that SSTC performance elicited a moderate level of exercise intensity of $53 \% \mathrm{VO}_{2}$ reserve [24]. Our study provides compelling evidence for the use of SSTC training as an alternative training modality in people with COPD.

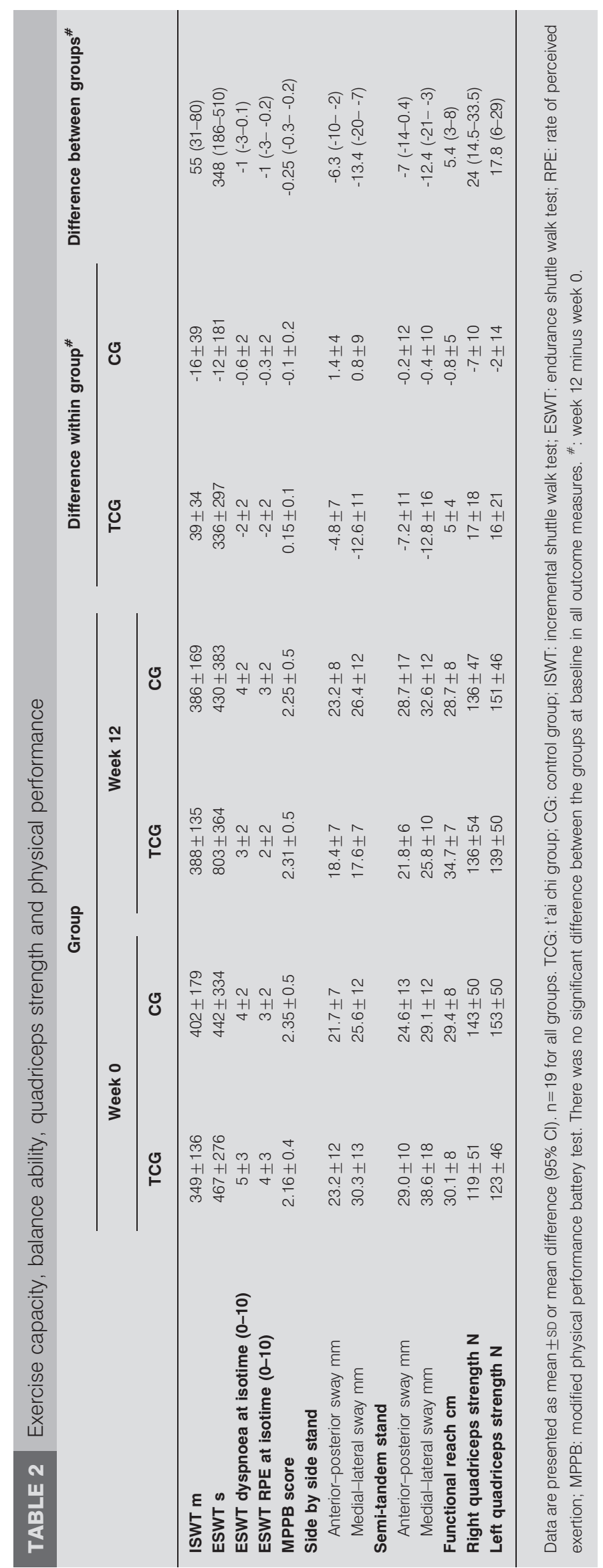


TABLE 3 Health related quality of life, anxiety and depression and self-efficacy

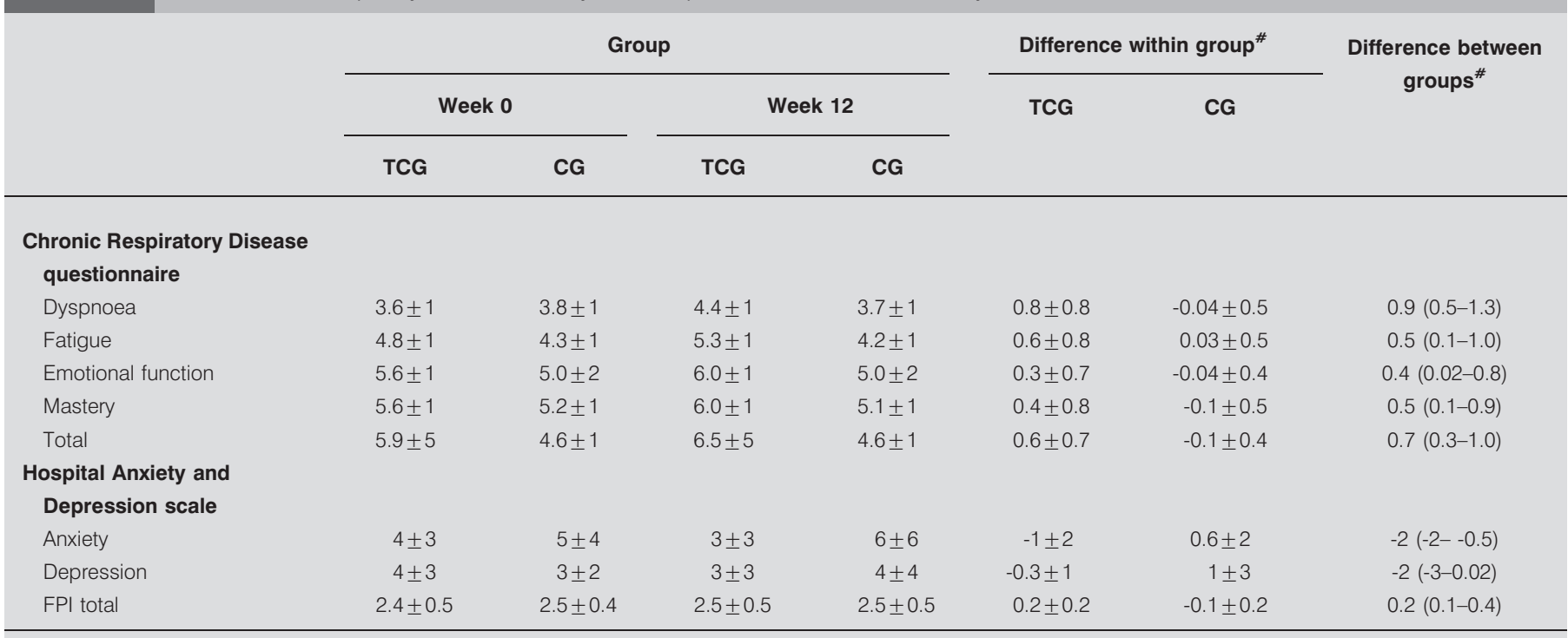

Data are presented as mean \pm SD or mean $(95 \% \mathrm{CI}) . n=19$ for all groups. TCG: t'ai chi group; CG: control group; FPI: Functional Performance Inventory. ${ }^{\#}$ : week 12 minus week 0.

The results of this study demonstrated that SSTC training has highly clinically relevant effects on endurance and peak exercise capacity in people with COPD as the improvements in ESWT and ISWT both exceeded the minimum clinically important difference (MID) which was developed based on improvements after a standard pulmonary rehabilitation programme (186 $\mathrm{s}$ for the ESWT and $47.5 \mathrm{~m}$ for the ISWT) $[25,26]$. This demonstrates that SSTC training can elicit similar improvements in exercise capacity to those achieved in standard pulmonary rehabilitation. In fact, the true effect of SSTC training was probably underestimated as six participants in the TCG reached the completion time of 20 min for the ESWT following training, with a potential to continue.

\begin{tabular}{|c|c|c|c|c|}
\hline \multirow[t]{2}{*}{ TABLE 4} & \multicolumn{4}{|c|}{$\begin{array}{l}\text { Physiological responses, oxygen saturation and } \\
\text { heart rate in incremental shuttle walk test (ISWT) } \\
\text { and short-form Sun-style t'ai chi performance }\end{array}$} \\
\hline & & ISWT rest & ISWT peak & T'ai chi \\
\hline $\mathrm{VO}_{2} \mathrm{~L} \cdot \mathrm{min}^{-1}$ & & $0.24 \pm 0.08$ & $1.07 \pm 0.28$ & $0.67 \pm 0.18$ \\
\hline$V \mathrm{CO}_{2} L \cdot \mathrm{min}^{-1}$ & & $0.22 \pm 0.09$ & $0.99 \pm 0.28$ & $0.57 \pm 0.17$ \\
\hline VT L & & $0.56 \pm 0.15$ & $1.18 \pm 0.32$ & $0.97 \pm 0.28$ \\
\hline$V E L \cdot \min ^{-1}$ & & $12.25 \pm 3.54$ & $36.41 \pm 7.61$ & $24.82 \pm 7.12$ \\
\hline fB breaths per & $\min$ & $23 \pm 5$ & $32 \pm 5$ & $27 \pm 6$ \\
\hline $\mathrm{SpO}_{2} \%$ & & $97 \pm 2$ & $91 \pm 7$ & $94 \pm 5$ \\
\hline $\begin{array}{l}\text { Heart rate beat } \\
\text { per min }\end{array}$ & & $81 \pm 13$ & $122 \pm 19$ & $105 \pm 17$ \\
\hline
\end{tabular}

Data presented as mean $\pm \mathrm{SD}$. ISWT rest: ISWT at rest; ISWT peak: ISWT end test; $\mathrm{VO}_{2}$ : oxygen consumption; $\mathrm{V}_{\mathrm{CO}_{2}}$ : carbon dioxide production; $\mathrm{VT}$ : tidal volume; $V E$ : minute ventilation; $\mathrm{fB}$ : breathing frequency; $\mathrm{SpO}_{2}$ : oxygen saturation.
While there have been no previous studies investigating the effect of SSTC in people with COPD, some other styles of $t^{\prime}$ ai chi have been investigated. A previous randomised controlled trial which investigated the effects of a t'ai chi programme, which emphasised breathing control compared to either unsupervised self-paced walking training or usual daily activity $[5,6]$, demonstrated a significant difference between groups, in favour of the t'ai chi group, in 6-min walk distance but not in the total score of the St George's Respiratory Questionnaire. In another study, YEH et al. [7] compared the effect of a simplified Yang-style $t^{\prime}$ ai chi programme (five forms) to usual medical care and showed that in the $t^{\prime}$ ai chi group there was a significant improvement in HRQoL but not in exercise capacity, self-efficacy or peak $\mathrm{VO}_{2}$ compared to usual care. The lack of effect of simplified $t^{\prime}$ ai chi in most outcomes might have been due to the small sample size (five participants in each group). In comparison, our study has stronger methodology with a larger sample size and only $9.5 \%$ drop-outs. The results of our study provide strong evidence for SSTC training over usual medical care to improve exercise capacity.

Impairments in balance and lower limb muscle strength are common in people with COPD $[27,28]$ and are some of the major risk factors for falls [29]. Interestingly, conventional pulmonary rehabilitation has not been shown to improve balance in people with COPD [30]. An important finding from our study was the significant improvement in balance and muscle strength following SSTC training, which has the potential to reduce the risk of falls in people with COPD.

An additional benefit of SSTC training was a significant improvement in HRQoL, anxiety and self-reported difficulty in functional performance compared to the control group. The change in CRQ scores exceeded the MID in nearly all domains in the TCG compared to the CG. This result suggested SSTC is 
a clinically relevant modality for improving HRQoL in people with COPD. The fact that exercise capacity and HRQoL showed similar improvements to those which can be achieved with pulmonary rehabilitation [31] suggest that t'ai chi training may be a reasonable alternative when comprehensive pulmonary rehabilitation is not available.

The high levels of compliance with supervised and unsupervised training during the SSTC training suggested that SSTC was a feasible training modality for people with COPD and could easily be practised as a home programme. Furthermore, the results of this study suggest that SSTC would be a suitable modality for people with multiple comorbidities, as comorbidities were common in this cohort (table 1).

A study in healthy populations has shown that $t^{\prime}$ ai chi achieves moderate exercise intensity [32]. However, the exercise intensity of $t^{\prime}$ ai chi has not been previously investigated in people with COPD; therefore, it is unknown whether the exercise intensity of $t^{\prime}$ ai chi is adequate to elicit physiological training benefits. Our study showed that the training intensity of SSTC reached a moderate level of $53 \%$ of $\mathrm{VO}_{2}$ reserve and such intensity met the minimal effective training intensity for improvement in cardiovascular fitness [24]. When the 15 participants from part B of the study (who were also in the TCG of part A) were compared to the CG, there was a significant increase in ESWT time, highlighting that the training intensity of SSTC resulted in an exercise training response.

\section{Limitations}

The use of wrist weights during SSTC performance is not standard practice. This limits the ability to generalise our reported intensity of SSTC. However, people with COPD are advised to exercise at a moderate level of dyspnoea or perceived exertion to elicit physiological training responses. Adding wrist weights is a feasible option to increase SSTC training intensity to achieve the appropriate levels of dyspnoea or perceived exertion.

\section{Conclusion}

In conclusion, SSTC was significantly more effective than no training in improving exercise capacity, balance, physical performance, quadriceps strength, HRQoL, anxiety and selfreported difficulty in functional performance in people with COPD. Compliance with both supervised and unsupervised training throughout the study was high. Furthermore, the intensity of SSTC exercise was moderate, which met the recommendation for exercise training in people with COPD. This study provides compelling evidence that $t^{\prime}$ ai chi training achieved an appropriate training intensity and that it may be an effective alternative training modality in people with COPD. With increasing numbers of people diagnosed with COPD, such an alternative training modality may provide more opportunities for exercise training.

\section{CLINICAL TRIAL}

This study is registered at www.anzct.org.au with identifier number ACTRN12608000383369.

\section{STATEMENT OF INTEREST}

Conflict of interest information can be found alongside the online version of this article at www.erj.ersjournals.com

\section{ACKNOWLEDGEMENTS}

The authors would like to acknowledge the Physiotherapy Research Foundation and the Ian Collier Memorial Scholarship for supporting this study, Samantha Ennis and Kathryn Skelsey (Lung and Heart research group, Faculty of Health Sciences, University of Sydney) for their assistance in assessing participants in this study and P. Lam (University of New South Wales, Sydney, Australia), R. Song (Chungnam National University, Daehangno, South Korea) and B. Roberts (University of Florida, Gainesville, USA) for reviewing the manuscript.

\section{REFERENCES}

1 Lan C, Lai JS, Chen SY, et al. Tai Chi Chuan to improve muscular strength and endurance in elderly individuals: a pilot study. Arch Phys Med Rehabil 2000; 81: 604-607.

2 Hong Y, Li JX, Robinson PD. Balance control, flexibility, and cardiorespiratory fitness among older Tai Chi practitioners. $\mathrm{Br} \mathrm{J}$ Sports Med 2000; 34: 29-34.

3 Yeh GY, McCarthy EP, Wayne PM, et al. Tai Chi exercise in patients with chronic heart failure. Arch Intern Med 2011; 171: 750757.

4 Fransen M, Nairn L, Winstanley J, et al. Physical activity for osteoarthritis management: a randomized controlled clinical trial evaluating hydrotherapy or Tai Chi classes. Arthritis Rheum 2007; 57: 407-414.

5 Chan AWK, Lee A, Suen LKP, et al. Tai Chi Qigong improves lung functions and activity tolerance in COPD clients: a single blind, randomized controlled trial. Complement Ther Med 2011; 19: 3-11.

6 Chan AWK, Lee A, Suen LKP, et al. Effectiveness of a Tai Chi Qigong program in promoting health-related quality of life and perceived social support in chronic obstructive pulmonary disease clients. Qual Life Res 2010; 19: 653-664.

7 Yeh GY, Roberts DH, Wayne PM, et al. Tai Chi exercise for patients with chronic obstructive pulmonary disease: a pilot study. Respir Care 2010; 55: 1475-1482.

8 Leung RWM, Alison JA, McKeough ZJ, et al. A study design to investigate the effect of short-form Sun-style Tai Chi in improving functional exercise capacity, physical performance, balance and health related quality of life in people with Chronic Obstructive Pulmonary Disease (COPD). Contemp Clin Trials 2011; 32: 267-272.

9 Celli BR, MacNee W. Standards for the diagnosis and treatment of patients with COPD: a summary of the ATS/ERS position paper. Eur Respir J 2004; 23: 932-946.

10 Borg GAV. Psychophysical bases of perceived exertion. Med Sci Sports Exerc 1982; 14: 377-381.

11 Quanjer PH, Tammeling GJ, Cote JE, et al. Lung volumes and forced ventilatory flows. Work group on standardisation of lung function tests, European Community for Steel and Coal. Official Statement of the European Respiratory Society. Eur Respir J 1993; 16: 5-40.

12 Singh SJ, Morgan MDL, Scott S, et al. Development of a shuttle walking test of disability in patients with chronic airways obstruction. Thorax 1992; 47: 1019-1024.

13 Revill SM, Morgan MD, Singh SJ, et al. The endurance shuttle walk: a new field test for the assessment of endurance capacity in chronic obstructive pulmonary disease. Thorax 1999; 54: 213-222.

14 Simonsick EM, Newman AB, Nevitt MC, et al. Measuring higher level physical function in well-functioning older adults: expanding familiar approaches in the Health ABC study. J Gerontol A Biol Sci Med Sci 2001; 56A: M644-M649. 
15 Lord SR, Menz HB, Tiedemann A. A physiological profile approach to falls risk assessment and prevention. Phys Ther 2003; 83: 237-252.

16 Duncan PW, Weiner DK, Chandler J, et al. Functional reach: a new clinical measure of balance. J Gerontol 1990; 45: M102-M197.

17 Guyatt GH, Berman LB, Townsend M, et al. A measure of quality of life for clinical trials in chronic lung disease. Thorax 1987; 42: 773-778.

18 Zigmond AS, Snaith RP. The hospital anxiety and depression scale. Acta Psychiatr Scand 1983; 67: 361-370.

19 Leidy NK. Functional performance in people with chronic obstructive pulmonary disease. Image J Nurs Sch 1995; 27: 23-34.

20 Casaburi R. Factors determining constant work rate exercise tolerance in COPD and their role in dictating the minimal clinically important difference in response to interventions. COPD 2005; 2: 131-136.

21 Sewell L, Singh SJ, Williams JEA, et al. How long should outpatient pulmonary rehabilitation be? A randomised controlled trial of 4 weeks versus 7 weeks. Thorax 2006; 61: 767-771.

22 Swain DP, Franklin BA. $\mathrm{VO}_{2}$ reserve and the minimal intensity for improving cardiorespiratory fitness. Med Sci Sports Exerc 2002; 34 152-157.

23 Luxton N, Alison JA, Wu J, et al. Relationship between field walking tests and incremental cycle ergometry in COPD. Respirology 2008; 13: 856-862.
24 Garber CE, Blissmer B, Deschenes MR, et al. Quantity and quality of exercise for developing and maintaining cardiorespiratory, musculoskeletal, and neuromotor fitness in apparently healthy adults: guidance for prescribing exercise. Med Sci Sports Exerc 2011; 43: 1334-1359.

25 Pepin V, Laviolette L, Brouillard C, et al. Significance of changes in endurance shuttle walking performance. Thorax 2010; 66: 115-120.

26 Watz H, Waschki B, Meyer T, et al. Physical activity in patients with COPD. Eur Respir J 2009; 33: 262-272.

27 Beauchamp MK, Lakhani B, Mathur S, et al. Impairments in systems underlying control of balance in COPD. Chest 2012; 141: 1496-1503.

28 Franssen FM, Broekhuizen R, Janssen PP, et al. Limb muscle dysfunction in COPD: effects of muscle wasting and exercise training. Med Sci Sports Exerc 2005; 37: 2-9.

29 Tinetti M, Speechley M, Ginter S. Risk factors for falls among elderly persons living in the community. N Engl J Med 1988; 319: 1701-1707.

30 Beauchamp MK, O'Hoski S, Goldstein RS. Effect of pulmonary rehabilitation in balance in persons with chronic obstructive pulmonary disease. Arch Phys Med Rehabil 2010; 91: 1460-1465.

31 Lacasse Y, Goldstein R, Lasserson TJ, et al. Pulmonary rehabilitation for chronic obstructive pulmonary disease. Cochrane Database Syst Rev 2006; CD003793.

32 Lan C, Chen SY, Lai JS, et al. Heart rate responses and oxygen consumption during Tai Chi Chuan practice. Am J Chinese Med 2001; 29: 403-410. 